\title{
Identification and characterization of a high kernel weight mutant induced by gamma radiation in wheat (Triticum aestivum L.)
}

Xuejiao Cheng ${ }^{1,2,3}$, Lingling Chai ${ }^{1,2,3}$, Zhaoyan Chen ${ }^{1,2,3}$, Lu Xu $^{1,2,3}$, Huijie Zhai ${ }^{1,2,3}$, Aiju Zhao ${ }^{4}$, Huiru Peng ${ }^{1,2,3}$, Yingyin $\mathrm{YaO}^{1,2,3}$, Mingshan You ${ }^{1,2,3}$, Qixin Sun ${ }^{1,2,3^{*}}$ and Zhongfu $\mathrm{Ni}^{1,2,3^{*}}$

\begin{abstract}
Background: Inducing mutations are considered to be an effective way to create novel genetic variations and hence novel agronomical traits in wheat. This study was conducted to assess the genetic differences between Shi4185 and its mutant line Fu4185, produced by gamma radiation with larger grain, and to identify quantitative trait loci (QTLs) for thousand kernel weight (TKW).

Results: Phenotypic analysis revealed that the TKW of Fu4185 was much higher than that of Shi4185 under five different environments. At the genomic level, 110 of 2019 (5.4\%) simple sequence repeats (SSR) markers showed polymorphism between Shi4185 and Fu4185. Notably, $30 \%$ (33 out of 110) polymorphic SSR markers were located on the D-genome, which was higher than the percentage of polymorphisms among natural allohexaploid wheat genotypes, indicating that mutations induced by gamma radiation could be a potential resource to enrich the genetic diversity of wheat D-genome. Moreover, one QTL, QTkw.cau-5D, located on chromosome 5DL, with Fu4185 contributing favorable alleles, was detected under different environments, especially under high temperature conditions.
\end{abstract}

Conclusions: QTkw.cau-5D is an environmental stable QTL, which may be a desired target for genetic improvement of wheat kernel weight.

Keywords: Wheat, Kernel weight, Mutant, SSR marker, QTL

\section{Background}

Bread wheat (Triticum aestivum L.) is one of the most important food crops, which accounts for $20 \%$ of the world's calorie consumption and growers (http://faostat3.fao.org/ home/E). With the ever-growing population in the world, a pressing objective is to increase wheat productivity, which could be dissected by improving wheat yield potential and raising the yield ceiling [1]. Wheat yield can be attributed to the integration of the number of fertile spikes per unit area, grains per spike, and the kernel weight. Among these three yield components, kernel weight is a

\footnotetext{
* Correspondence: qxsun@cau.edu.cn; nizf@cau.edu.cn

'State Key Laboratory for Agrobiotechnology and Key Laboratory of Crop Heterosis and Utilization (MOE) and Key Laboratory of Crop Genomics and Genetic Improvement (MOA), Beijing Key Laboratory of Crop Genetic Improvement, China Agricultural University, Yuanmingyuan Xi Road No. 2, Haidian District, Beijing 100193, China

Full list of author information is available at the end of the article
}

highly heritable trait and has made significant contributions to yield potential in modern wheat breeding [2]. For example, TKW of Chinese wheat varieties has increased by 2.19 g every 10 years from 1940 to 2000 [3]. However, for the majority of the $20^{\text {th }}$ century, TKW was mainly improved by applying selection techniques over phenotypic measurements. These years, detection of QTLs with molecular markers has drawn more attention. Up to date, QTLs for TKW have been mapped on all 21 wheat chromosomes [4-9]. In addition, the grain size-controlling genes in rice showed a significant association with the orthologs in wheat for the TKW trait [10-12].

Increasing temperature is a major element of observed global climate change (https://www.ncdc.noaa.gov/indicators/). For wheat, global production is estimated to fall by $6 \%$ for each ${ }^{\circ} \mathrm{C}$ of further temperature increase [13]. Thus, high temperature (heat) stress during grain filling 
becomes a major problem for almost all wheat production areas in temperate regions $[14,15]$ and developing heat-tolerant cultivars has become an important objective for breeders. Extensive research into the heat tolerance of wheat during grain filling revealed that kernel weight was more suited for screening than other traits evaluated [16-19]. Therefore, identification of DNA markers associated with kernel weight under post-anthesis high temperature stress would allow marker-assisted selection (MAS) and increase the efficiency for improving yield potential during breeding. Despite its importance, only a few QTL mapping studies of kernel weight have focused on heat stress.

A powerful approach for deciphering the biological functions of genes is to produce mutants with altered phenotypes or physiological responses. In wheat, chemical and ionizing radiation mutagenesis have been universally used to generate genetic variations for breeding researches and genetic studies. In total, 274 mutant varieties of wheat were developed by physical or chemical mutagens from 1930 to 2014 (http://www-naweb.iaea.org/nafa/pbg/). As a mutant induced by gamma radiation from Shi4185, Fu4185 showed higher kernel weight. This study aimed to analyze the genetic differences between Fu4185 and Shi4185 by SSR markers and to identify the genomic regions responsible for kernel weight in segregating populations derived from Shi4185 and Fu4185 under timely and late-sown conditions at different locations. This study will contribute to a better understanding of the genome-wide genetic variation and the stability of the QTL for kernel weight under heat stress.

\section{Methods}

\section{Plant materials and field experiment}

Shi4185 is an elite wheat cultivar in the Northern China winter wheat region. Fu4185 is a gamma radiationinduced mutant of advanced generations (M8), which was donated by Dr. Fengwu Zhao (Hebei Academy of Agricultural Sciences, China). $F_{2}$ seeds were generated by self-pollinating of the $F_{1}$ progeny of Shi4185 and Fu4185 in 2010. A total of $249 \mathrm{~F}_{2}$ individuals and the two parental lines were planted in the experimental station at Shangzhuang, Beijing $\left(40^{\circ} 06^{\prime} \mathrm{N}, 116^{\circ} 11^{\prime} \mathrm{E}\right)$ in the autumn of 2011 (BJ-2011), and their derived $F_{2: 3}$ lines were grown at Gaoyi, Hebei $\left(37^{\circ} 37^{\prime} \mathrm{N}, 114^{\circ} 35^{\prime} \mathrm{E}\right)$ (HB-2012) and Linfen, Shanxi $\left(36^{\circ} 05^{\prime} \mathrm{N}, 111^{\circ} 30^{\prime} \mathrm{E}\right)$ (SX-2012) in the autumn of 2012 with three replicates. For spring sowing, the seeds of $\mathrm{F}_{2}$ populations were surface sterilized with $70 \%$ ethanol for $30 \mathrm{~s}$ and $10 \% \mathrm{NaClO}$ for $10 \mathrm{~min}$, and vernalized for 40 days in Petri dishes on wet filter paper at $4{ }^{\circ} \mathrm{C}$ in the dark after germination. After that, the seeds of two $\mathrm{F}_{2}$ populations were planted in BJ on February 17, 2014 (BJ-2014) and in SX on March 1, 2014 (SX-2014), respectively. The $F_{2}$ seeds were space-planted $\left(7.5 \mathrm{~cm}\right.$ between plants), and each $\mathrm{F}_{2: 3}$ line was hand-sown using a randomized complete block design in two-row plots of $1.5 \mathrm{~m}$ long row with $0.3 \mathrm{~m}$ spacing between the rows.

\section{Trait evaluation}

Data of yield and yield contributing traits (plant height, spike length, spikelet number per spike, thousand kernel weight) of Shi4185 and Fu4185 that were planted at BJ in the autumn of 2011 were recorded using 30 plants (10 random plants from each replication) before harvest. After harvest, TKW of $\mathrm{F}_{2}$ individuals were determined. For the $249 \mathrm{~F}_{2: 3}$ lines, bulked seeds of 10 random plants from the same line per replication were measured for TKW using a camera-assisted phenotyping system. This system was provided by Hangzhou Wanshen Detection Technology Co., Ltd. (Hangzhou, China).

\section{DNA extraction and SSR marker analysis}

Genomic DNA was extracted from seedling young leaves using the protocol as described by Sharp et al. [20]. The DNA was precipitated again with isopropanol, washed twice with $70 \%$ ethanol and dissolved in TE buffer. DNA quality was checked using $1 \%$ agarose to make sure no noticeable degradation.

The parental lines were screened with a total of 2019 SSR markers (gwm, wmc, barc, $c f d, k s m, g d m$ and $p k$ ). Primer sequences for most SSR markers are available at http://wheat.pw.usda.gov/GG2/index.shtml. Primer sequences for SSR markers designed in our lab are partially listed in Table 1. PCR amplifications were carried out in a $10 \mu \mathrm{L}$ volume containing $40 \mathrm{ng}$ genomic DNA, $1 \mu \mathrm{L} 10 \times$ reaction buffer, $0.2 \mu \mathrm{L} 10 \mathrm{mmol} \mathrm{L}^{-1} \mathrm{dNTPs}$, $1.0 \mu \mathrm{L}$ primer, $1 \mathrm{U}$ rTaq DNA polymerase (Takara, Dalian) and $5.7 \mu \mathrm{L} d d \mathrm{H}_{2} \mathrm{O}$. The PCR was performed by initially denaturing the template DNA at $94{ }^{\circ} \mathrm{C}$ for $5 \mathrm{~min}$, followed by 35 cycles at $94{ }^{\circ} \mathrm{C}$ for $45 \mathrm{~s}, 55^{\circ} \mathrm{C}$ for $30 \mathrm{~s}$, and $72{ }^{\circ} \mathrm{C}$ for $30 \mathrm{~s}$, then terminated by a final extension for $10 \mathrm{~min}$ at $72{ }^{\circ} \mathrm{C}$. PCR fragments were separated on $8 \%$ non-denatured polyacrylamide gel electrophoresis (PAGE) and visualized by silver staining according to Marklund, Chaudhary et al. [21].

\section{Marker development and QTL analysis}

International Wheat Genome Sequencing Consortium (IWGSC) (http://www.wheatgenome.org/) has published survey sequence assemblies of the 21 individual chromosomes of Chinese Spring (Triticum aestivum L.). Firstly, the SSR markers were developed by using the survey sequence assembly of chromosome 5DL. SSR markers were designed under the conditions that dinucleotide and trinucleotide repeats were more than 30 and 21 times, respectively, in the SSR-flanking regions. Secondly, Aegilops tauschii is the diploid progenitor of the D-genome of 
Table 1 Polymorphic SSR markers for genetic map construction

\begin{tabular}{llll}
\hline Markers & Forward primer $\left(5^{\prime}-3^{\prime}\right)$ & Reverse primer $\left(5^{\prime}-3^{\prime}\right)$ & Annealing temperature $\left({ }^{\circ} \mathrm{C}\right)$ \\
\hline Xcau1022 & CCTAACCATCCAACCATAAGT & TTCACGTACTCAAAAGTGG & 55 \\
Xcau1053 & GGATGTACATTGAACAGTGCT & CCCACCCTACTCACTTCAA & 55 \\
Xcau1074 & ACCTAAAATCTTCCCCCTACT & GCCAATTAATGCAGACTAGC & 55 \\
Xcau1087 & GGAAATCATGCACACATGG & CCCCACCCTACTCACTTCAA & 58 \\
Xcau1118 & TGTAATCCGTCTCCTACCTTAT & GTCATACATGTCATCGGACTAC & 55 \\
Xcau1132 & AGTCAATGAACAGAGCCATC & CTTGACCTAGATAGGGAACAA & 55 \\
\hline
\end{tabular}

allohexaploid wheat, and its genome is an invaluable reference for wheat genomics [22]. Recently, the single nucleotide polymorphism (SNP) genetic map of Aegilops tauschii, was constructed, and the collinearity between Brachypodium, rice, and sorghum was established [23]. Therefore, comparative genomics approaches were employed to design SSR markers using the draft genome sequences, the extended SNP marker sequences and BAC scaffolds of Aegilops tauschii accession AL8/78. Information of polymorphic SSR markers for genetic map construction was listed in Table 1.

Linkage analysis of the marker was performed using JoinMap 4.0 software (http://www.kyazma.nl/index.php/ mc.JoinMap/sc.General). According to the linkage distance between markers, a linkage map was drawn using MapChart v2.2 software (http://www.biometris.nl). Single marker analysis and QTL analysis by Composite interval mapping (CIM) method were performed by WinQTL Cart 2.5 software package [24]. The logarithm of the odds (LOD) threshold scores were calculated using 1,000 permutations [25], [26]. A QTL was declared when the LOD score was greater than 2.0.

\section{Results}

\section{Phenotypic analysis}

To characterize the phenotypic difference between Shi4185 and Fu4185, these two genotypes were firstly planted in BJ in the autumn of 2011 for analysis. As shown in Fig. 1, Shi4185 and Fu4185 showed significant differences in spike length and kernel size, whereas no difference was detected for plant height and spikelet number per spike. As expected, the TKW of Fu4185 was higher than that of Shi4185, which exhibited a good reproducibility in different environments (Table 2). In addition, there was a wide range of variations for both $\mathrm{F}_{2}$ and $\mathrm{F}_{2: 3}$ populations, with coefficients of variation (CVs) ranging from 9.59 to $11.86 \%$ in the $\mathrm{F}_{2}$ populations and from 4.0 to $4.87 \%$ in the $\mathrm{F}_{2: 3}$ populations, respectively. All populations showed continuous distributions with transgressive segregation on both sides for TKW (Additional file 1). Notably, the calculated broad-

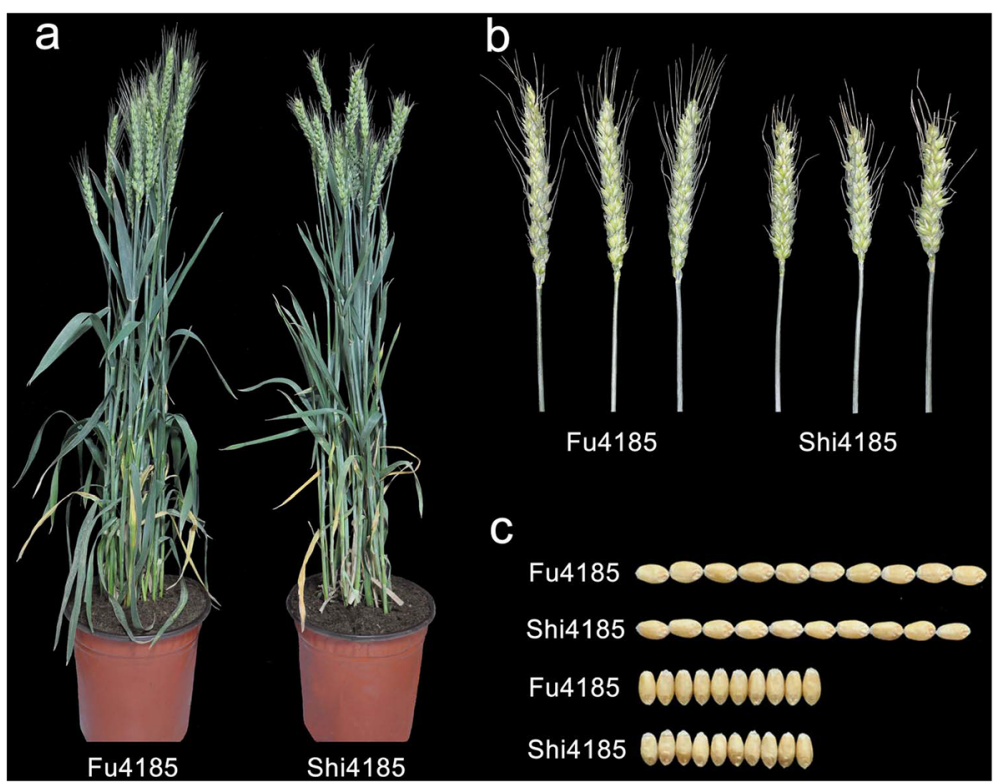

Fig. 1 Phenotypic comparison of Shi4185 and Fu4185 planted at BJ-2011. a Plants at the filling stage; b Spikes at the filling stage; c Kernels after harvest 
Table 2 TKW (g) of Shi4185 and Fu4185 in different environments

\begin{tabular}{llll}
\hline Environment & Sowing season & Shi4185 & Fu4185 \\
\hline BJ-2011 & Autumn & $36.01 \pm 0.86$ & $42.82 \pm 0.75^{* *}$ \\
HB-2012 & Autumn & $33.51 \pm 1.29$ & $37.14 \pm 1.10^{*}$ \\
SX-2012 & Autumn & $32.23 \pm 1.26$ & $39.44 \pm 0.70^{* *}$ \\
BJ-2014 & Spring & $33.76 \pm 1.09$ & $38.79 \pm 1.20^{* *}$ \\
SX-2014 & Spring & $29.90 \pm 1.10$ & $36.61 \pm 1.41^{* *}$ \\
\hline
\end{tabular}

*Indicates a significance level of $P \leq 0.05$; **Indicates a significance level of $P \leq 0.01$. All data are given as mean \pm SD

sense heritability $\left(h_{B}^{2}\right)$ of TKW based on the data of $F_{2: 3}$ populations in HB-2012 and SX-2012 were $88.5 \%$ and $86.9 \%$, respectively, suggesting that TKW is suitable for QTL mapping (Table 3).

\section{Genetic variations between Shi4185 and Fu4185}

To investigate the genetic variations between Shi4185 and Fu4185, a total of 2019 SSR markers were used for analysis and 110 (5.4\%) markers were found to be polymorphic between Shi4185 and Fu4185. Two types of polymorphism were observed: presence or absence of the fragment (Type 1), and length differences of the fragment (Type 2). The numbers of the markers belong to Type 1 and 2 were 34 (30.9\%) and 76 (69.1\%), respectively. In addition, among the 110 polymorphic markers, 105 were located on 20 wheat chromosomes, with the exception of $4 \mathrm{~B}$. The numbers of polymorphic markers on each chromosome ranged from 1 (1A) to $14(2 B)$.

\section{Single-marker analysis}

To identify molecular markers associated with TKW, a total of $249 \mathrm{~F}_{2}$ individuals, planted at BJ in the autumn of 2011, were genotyped using 58 polymorphic SSR markers. After the genotypic data of the $F_{2}$ population was merged with the phenotypic data of the $F_{2}$ and $F_{2: 3}$ populations, respectively, single-marker analyses were conducted using WinQTLCart version 2.5. The results showed that marker Xbarc239 was significantly associated with TKW in both the $F_{2}$ and the $F_{2: 3}$ populations at two different locations, whereas marker Xgwm68 was

Table 3 TKW (g) of $F_{2}$ and $F_{2: 3}$ populations in different environments

\begin{tabular}{llllll}
\hline Environment & Population & Range & Mean & SD & $h_{B}^{2}(\%)$ \\
\hline BJ-2011 & $F_{2}$ & $25.80-54.43$ & 40.83 & 4.84 & - \\
HB-2012 & $F_{2: 3}$ & $32.85-40.59$ & 36.24 & 1.45 & 86.9 \\
SX-2012 & $F_{2: 3}$ & $31.56-39.85$ & 35.83 & 1.75 & 88.4 \\
BJ-2014 & $F_{2}$ & $20.55-47.07$ & 37.56 & 3.67 & - \\
SX-2014 & $F_{2}$ & $23.64-44.05$ & 34.60 & 3.32 & - \\
\hline
\end{tabular}

-Indicates no data only significantly linked with TKW in the $F_{2: 3}$ populations (Table 4).

The genetic distance between Xbarc239 and Xgwm68 was $36.6 \mathrm{cM}$. Previous studies reported that Xbarc239 was located on chromosome 5DL [27]. To verify the chromosome location of Xbarc239, the amplified products of the parental lines were cloned into the pEASY-blunt Simple Cloning Vector (Transgen) for sequencing and blasted against the wheat chromosome survey sequence database (http://www.wheatgenome.org/). The results showed that the amplified products exhibited much higher similarity (98.6\%) to chr5DL_ab_k71_contigs_4520158 as compared with chr5AL_ab_k95_contigs_1_2810952 and chr5BL_ab_ k71_contigs_10879865 (56.1\% and $66.7 \%$, respectively), suggesting that Xbarc239 was located on chromosome 5DL (Fig. 2, Additional file 2).

\section{Genetic linkage map construction of chromosome 5DL and QTL mapping}

To further explore polymorphic markers within the QTL region of TKW on chromosome 5DL, 137 SSR markers were developed using the wheat survey assembly sequence and the draft genome sequences of Aegilops tauschii, among which 26 markers showed polymorphism between Shi4185 and Fu4185. These polymorphic markers were used for linkage map construction by genotyping 249 individuals of the $F_{2}$ population (BJ-2011). Finally, one genetic linkage map of chromosome 5DL was constructed, including 8 SSR markers and spanning a total length of 84.96 cM (Fig. 3).

For QTL mapping, the genotype data of $\mathrm{F}_{2}$ population were merged with the phenotype data of both $F_{2}$ and two $\mathrm{F}_{2: 3}$ populations, respectively. Though composite interval mapping, one QTL for TKW (QTkw.cau-5D) was detected besides Xbarc239, with Fu4185 contributing favorable alleles (Fig. 3). For $F_{2}$ population, the Fu4185 allele at QTkw.cau-5D increased TKW by $2.65 \mathrm{~g}$, explaining $10.61 \%$ of the phenotypic variation. For the two $\mathrm{F}_{2: 3}$ populations, the Fu4185 allele at QTkw.cau-5D increased TKW by 0.45 to $0.56 \mathrm{~g}$, explaining 1.58 to $2.94 \%$ of the phenotypic variation (Table 5).

To further assess the environmental stability of QTkw.cau-5D, another two $\mathrm{F}_{2}$ populations derived from Fu4185 and Shi4185 were grown at two locations during the spring season of 2014. Four SSR markers (Xcau1053, Xcau1087, Xbarc239 and Xcau1118) were used to genotype the two populations. Genetic linkage map construction and composite interval mapping were carried out for each $F_{2}$ populations. QTL mapping analysis showed that QTkw.cau-5D could be detected in both the two populations, with Fu4185 contributing favorable alleles. The additive effects ranged from 1.43 to $1.70 \mathrm{~g}$, explaining 9.51 to $14.87 \%$ of the phenotypic variation (Table 5). 
Table 4 Single marker analysis of TKW in $F_{2}$ and $F_{2: 3}$ populations

\begin{tabular}{lllllcll}
\hline Environment & Population & marker & b0 & b1 & $-2 \ln (\mathrm{LO} / \mathrm{L} 1)$ & $\mathrm{F}(1, n-2)$ & $\operatorname{Pr}(\mathrm{F})$ \\
\hline BJ-2011 & $\mathrm{F}_{2}$ & Xbarc239 & 40.94 & -2.0 & 21.27 & 22.03 & $0.0000^{* * * *}$ \\
HB-2012 & $F_{2: 3}$ & Xbarc239 & 36.28 & -0.68 & 27.55 & 28.90 & $0.000^{* * * *}$ \\
& & Xgwm68 & 36.26 & -0.74 & 30.89 & 32.63 & $0.000^{* * * *}$ \\
SX-2012 & $F_{2: 3}$ & Xbarc239 & 35.87 & -0.73 & 21.68 & 22.47 & $0.000^{* * * *}$ \\
& & Xgwm68 & 35.84 & -0.55 & 11.33 & 11.49 & $0.001^{* * *}$ \\
\hline
\end{tabular}

***Indicates a significance level of $P \leq 0.001 ;{ }^{* * * *}$ Indicates a significance level of $P \leq 0.0001$

\section{Discussions}

Frequency, pattern and chromosome location of mutation at microsatellite loci between Shi4185 and Fu4185

Microsatellites or SSRs are composed of tandemly repeated, simple DNA sequence motifs of 1-6 nucleotide bases in length. Microsatellite markers are important tools for plant breeding, genetic and evolution studies [28]. A number of studies in animals and plants have calculated the natural mutation rate of microsatellites, and showed that the mutation rate varies greatly among species, ranging from $5 \times 10^{-6}$ in Drosophila [29-31] to $10^{-3}$ in human $[32,33]$. Gamma radiation can cause different types of mutations such as deletions, insertions, inversions and single-base substitution [34], but few studies have analyzed the frequency, polymorphism and pattern of microsatellite between the mutants and wild types in plants. In this study, SSR markers were employed to calculate the mutation frequencies induced by gamma radiation. Among the 2019 SSR markers, 110 (5.4\%) showed polymorphism between Shi4185 and Fu4185, which is lower than the percentage of polymorphism among natural allohexaploid wheat genotypes [35], but higher than the natural mutation rate of microsatellites per generation in different species [36]. In addition, two types of polymorphism were observed for SSR markers, including presence or absence of fragments (type 1) and fragment length differences for SSR markers (type 2). Multiple factors contribute to the observed variations. Type 1 might be produced by deletions, insertions, inversions and point mutations in the primer binding regions, whereas type 2 might be caused by insertion or deletion of bases in the SSR repeat regions. Consistent with this hypothesis, we sequenced marker Xbarc239 and this mutation proved to be a change in the number of TTA repeats.

The knowledge of chromosome location for polymorphic SSR markers between Shi4185 and Fu4185 is critical for the mutation research, because it could enable us to relate the SSR loci with the altered phenotypes. Our data showed that 105 of 110 polymorphic SSR markers were distributed on 20 chromosomes (except 4B). The numbers of polymorphic markers on each chromosome ranged from 1 (1A) to 14 (2B), suggesting that mutation frequency are not uniform across the genome. Although gamma radiation induced a large number of mutations on the genome, only a few agronomical traits showed differences between Fu4185and Shi4185. Moreover, only 2 of 110 polymorphic SSR markers were significantly associated with TKW. Collectively, we speculated that most mutation events may not result in noticeable phenotypic changes, indicating that the wheat genome could largely bear the mutations.

Hexaploid common wheat (Triticum aestivum L.; genome AABBDD) evolved by natural hybridization of emmer wheat (Triticum turgidum L.; genome AABB) and Aegilops tauschii Coss. (genome DD) [37, 38]. Only a few Aegilops tauschii's intraspecific lineages contributed to the evolution of common wheat, which resulted in relatively narrow genetic variation on the D-genome in wheat [39-42].

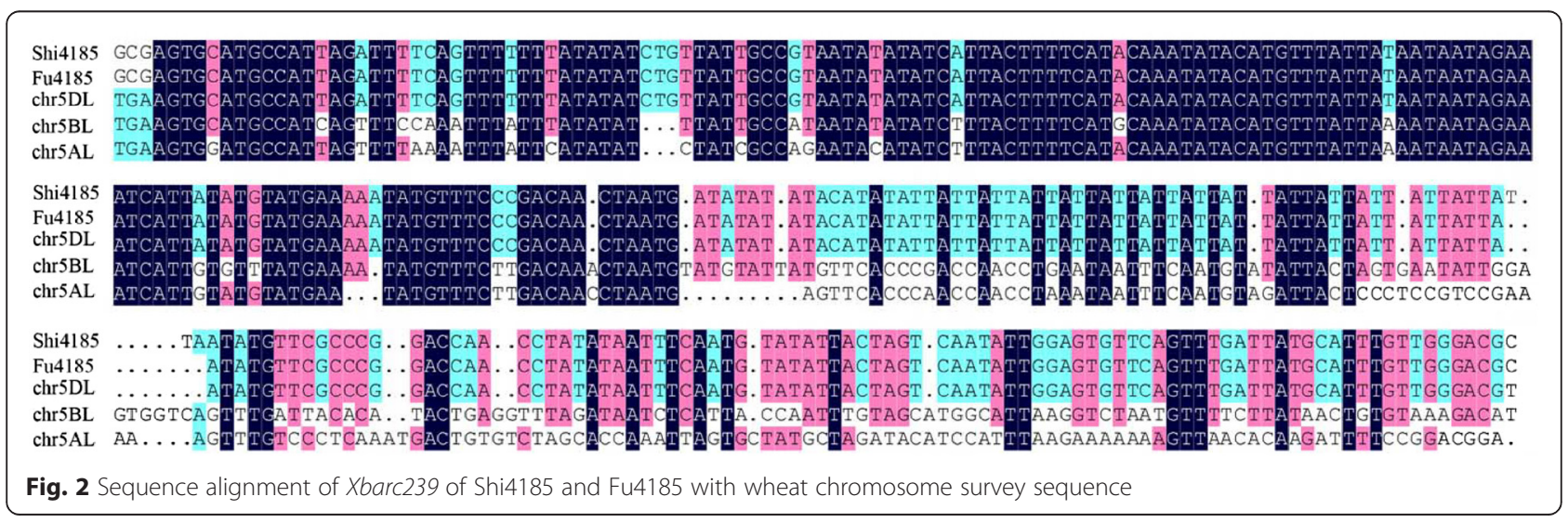




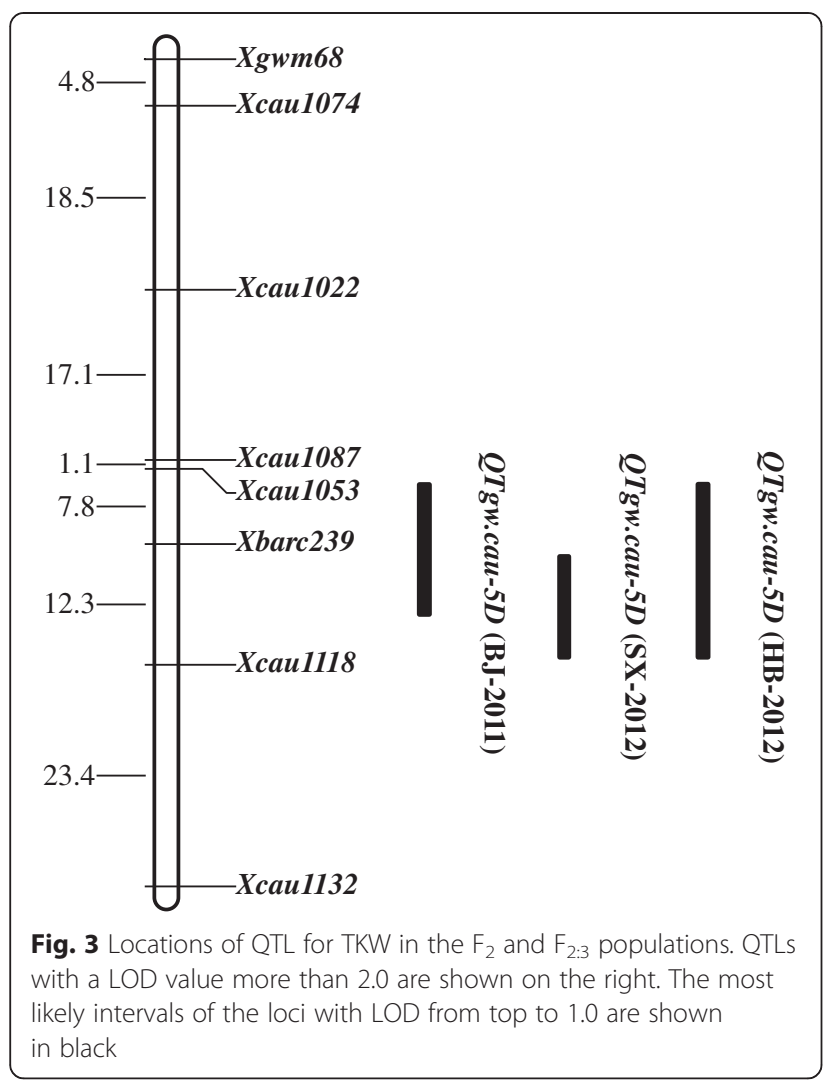

Consistently, the average genetic diversity of D-genome in bread wheat was lower than that of Aegilops tauschii [43]. Interestingly, we found that 33 of 110 polymorphic SSR markers were located on D-genome. Moreover, the QTL of kernel weight was mapped on chromosome 5DL, with Fu4185 contributing favorable alleles. Taken together, we proposed that mutants induced by gamma radiation could be a potential resource to enrich the genetic diversity of wheat D-genome.

\section{Environmental stability of QTL on chromosome 5DL controlling wheat kernel weight}

Kernel weight is a complex quantitative and important agronomic trait. Mapping QTLs is the preliminary work for the genetic improvement by MAS [44]. To date, many studies have identified QTLs controlling kernel weight in common wheat cultivars and the QTLs were assigned to various chromosomes [4-9]. For example, Simmonds et al. [45] reported that the effect of yield QTL, located on chromosome 6A, was driven primarily by increased kernel weight due to wider grains, indicating that the enhancement of kernel weight by MAS may benefit the genetic improvement of wheat yield. In this study, QTL mapping for TKW was conducted using $\mathrm{F}_{2}$ and $F_{2: 3}$ populations derived from Shi4185 and Fu4185. One major QTL (QTkw.cau-5D), with Fu4185 contributing favorable alleles, was consistently detected on chromosome 5DL under different environments, which was linked to SSR marker Xbarc239. However, phenotypic variation explained by $Q T k w . c a u-5 D$ was not very stable under different environments, with a range of 1.58 to $14.87 \%$. Moreover, the effect of QTkw.cau-5D in $\mathrm{F}_{2: 3}$ were smaller than in $F_{2}$ populations. These results may be partially explained by the following reasons: 1) Effects of other QTLs that were not detected; 2) Inaccurate phenotypic data of each genotype in $F_{2}$ populations for quantitative trait; 3) Impact of severe weather on TKW of $F_{2: 3}$ populations. According to the weather record, we speculated that excessive rainfall at the late stage of wheat grain filling in HB-2012 and SX-2012 may result in the smaller effect of QTkw.cau-5D in $\mathrm{F}_{2: 3}$ as compared to $\mathrm{F}_{2}$ populations.

Heat stress is one of key abiotic stress affecting wheat production in temperate regions [14, 15]. In traditional breeding, membrane thermostability and chlorophyll fluorescence were used as indicators of heat-stress tolerance in wheat, as they showed strong genetic correlation with grain yield, but the method was a time and laborconsuming process [46]. Mapping QTLs linked to heat stress tolerance traits will help to develop wheat cultivars suitable for high-temperature environments through MAS strategy. Recently, different traits like grain filling duration (GFD), thousand kernel weight (TKW) and yield have proved to be the preferable criteria to screen for heat-tolerant wheat in fields and many QTLs with significant effects on heat tolerance have been detected $[17,18,47]$. QTLs can be categorized according to the stability of their effects across environmental conditions: a "constitutive" QTL is consistently detected across most

Table 5 Additive effects of QTL for TKW in $F_{2}$ and $F_{2: 3}$ populations

\begin{tabular}{|c|c|c|c|c|c|c|}
\hline Environment & Population & Marker interval ${ }^{a}$ & Position $(\mathrm{CM})^{\mathrm{b}}$ & LOD & Additive effect $^{c}$ & Contribution (\%) \\
\hline BJ-2011 & $\mathrm{F}_{2}$ & Xcau1053-Xbarc239 & 6.01 & 5.23 & -2.65 & 10.61 \\
\hline HB-2012 & $F_{2: 3}$ & Xcau1053-Xbarc239 & 7.81 & 2.28 & -0.45 & 2.94 \\
\hline SX-2012 & $F_{2: 3}$ & Xbarc239-Xbarc1118 & 5.98 & 2.27 & -0.56 & 1.58 \\
\hline BJ-2014 & $\mathrm{F}_{2}$ & Xcau1087-Xbarc239 & 5.05 & 4.97 & -1.70 & 9.51 \\
\hline SX-2014 & $\mathrm{F}_{2}$ & Xcau1053-Xbarc239 & 0.00 & 2.58 & -1.43 & 14.87 \\
\hline
\end{tabular}

${ }^{a}$ Marker interval is the interval containing the significant peak value of the QTL

${ }^{\mathrm{b}}$ Position means the distance of the significant peak value for the QTL from the first marker in the marker interval

${ }^{\mathrm{C}} \mathrm{A}$ positive value indicates the Fu4185 allele having a positive effect on TKW, and negative value indicates Shi4185 allele having positive effect on TKW 
Table 6 List of environment, flowering date, maturity date and No. of days for different daily maximum temperature ${ }^{\text {a }}$ (Tmax) during grain filling

\begin{tabular}{|c|c|c|c|c|c|c|}
\hline \multirow[t]{2}{*}{ Environment } & \multirow{2}{*}{$\begin{array}{l}\text { Flowering } \\
\text { date }\end{array}$} & \multirow[t]{2}{*}{ Maturity date } & \multicolumn{4}{|c|}{ No. of days for different Tmax during grain filling } \\
\hline & & & $<30^{\circ} \mathrm{C}$ & $30-34{ }^{\circ} \mathrm{C}$ & $>35^{\circ} \mathrm{C}$ & Sum \\
\hline BJ-2011 & May 5, 2012 & June 13, 2012 & 24 & 16 & 0 & 40 \\
\hline HB-2012 & May 1, 2013 & June 5, 2013 & 21 & 12 & 2 & 35 \\
\hline SX-2012 & May 5, 2013 & June 9, 2013 & 18 & 13 & 5 & 36 \\
\hline BJ-2014 & May 11, 2014 & June 20, 2014 & 16 & 21 & 4 & 41 \\
\hline SX-2014 & May 15, 2014 & June 25, 2014 & 9 & 29 & 4 & 42 \\
\hline
\end{tabular}

${ }^{\mathrm{a}}$ Data of Tmax from individual year of each environment (http://www.tianqi.com/)

environments; an "adaptive" QTL is detected only under specific environmental conditions [48, 49]. An important prerequisite for a successful MAS program aimed at improving heat tolerance is the identification of the "constitutive" QTLs. Recently, timely and late-sown were used for mapping QTLs associated with heat tolerance in wheat and two "constitutive" QTLs were detected on chromosome $2 \mathrm{~B}$ and $7 \mathrm{~B}$ [18]. Applying this method, we tested the effects of QTkw.cau-5D under different environments. Although the number of the days with the maximum temperature above $30{ }^{\circ} \mathrm{C}$ from flowering to harvesting at spring sowing season was much more than that at autumn sowing season (Table 6), QTkw.cau-5D was also detected under high temperature environments, which provide further evidence that it is an environmental stable QTL. However, to determine the accurate effect of QTkw.cau$5 D$ under heat stress, it is better to compare spring and autumn planted trials in the same year, which deserved for further investigation.

In this study, the marker Xbarc239 linked to QTkw.cau$5 D$ was physically located to the bin of 5DL1-0.60-0.74 using Chinese Spring deletion lines [27]. When comparing previous results [50-53], we found that QTkw.cau-5D was located to the same bin as the QTL for TKW between Xswes340a and Xswes342b (Xgwm174) [51] (Fig. 4). Although further analysis was needed to clarify the relationship of these two QTLs, QTkw.cau-5D detected in our study may be a more desired target for genetic

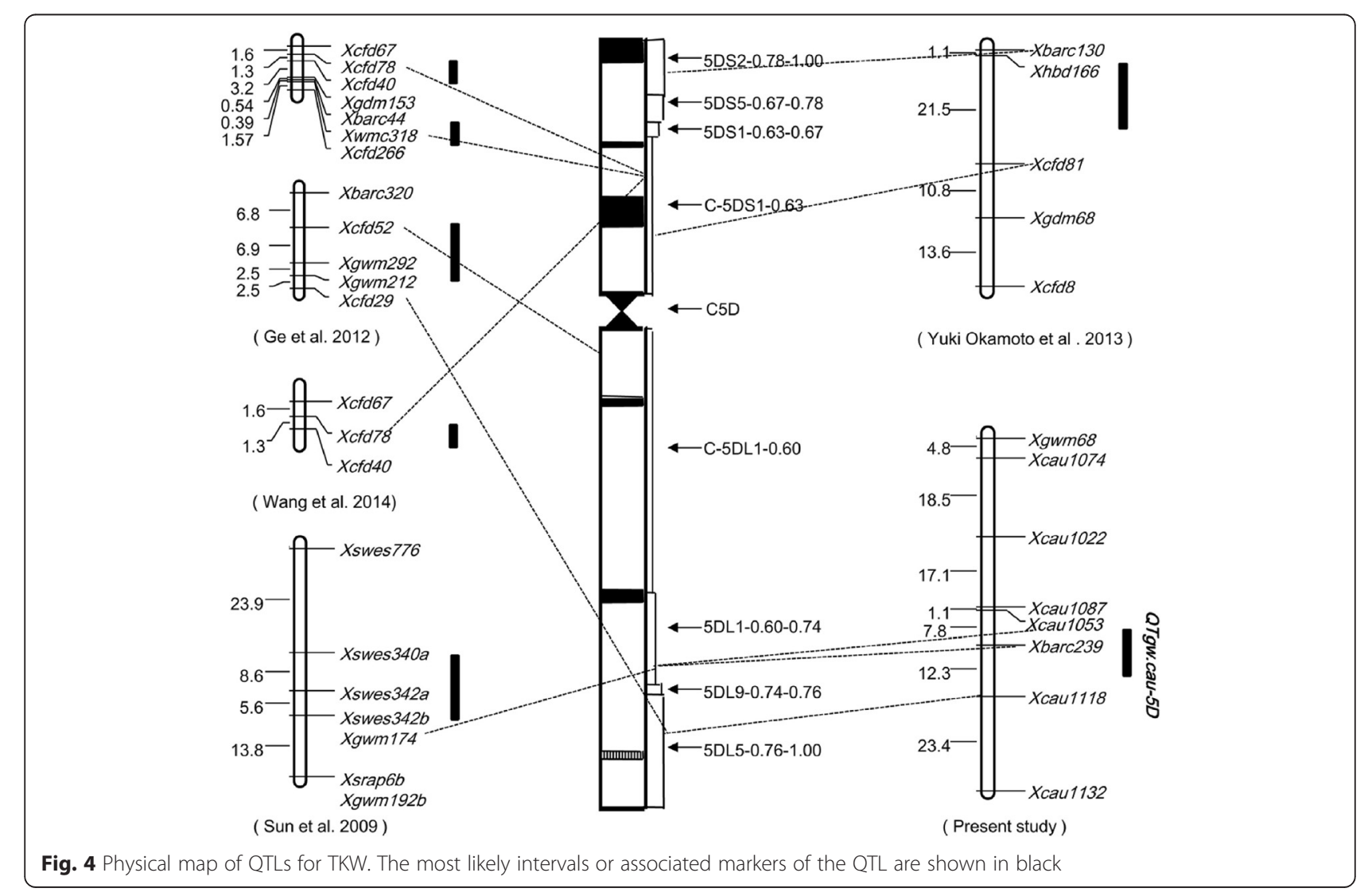


improvement of wheat kernel weight. Firstly, QTkw.cau$5 D$ is an environmental stable QTL, whereas the QTL on chromosome 5D reported by Sun et al. [51] was only detected in 2 of 4 experimental environments; Secondly, the donor parent of Fu4185 (Shi4185) is an elite wheat cultivar in the Northern China winter wheat region. Moreover, a number of new cultivars derived from Shi4185 have been released in China, such as Henong130, Baomai10 and Nongda399. Theoretically, Fu4185 is a potential elite parent for genetic improvement of kernel weight in wheat breeding; Finally, considering the severe effect of heat stress on kernel weight in wheat production, our identified QTL associated with kernel weight under high temperature stress may increase the efficiency for improving wheat yield potential by MAS.

\section{Conclusions}

One favorable and environmental stable QTL allele on chromosome 5DL controlling kernel weight was identified in Fu4185, a mutant of an elite wheat cultivar Shi4185 induced by gamma radiation. Furthermore, 30 \% (33 of 110) polymorphic SSR markers between Shi4185 and Fu4185 were located on the D-genome. Taken together, these data revealed that mutations induced by gamma radiation could be a potential resource to enrich the genetic diversity of wheat D-genome.

\section{Additional files}

Additional file 1: The data of genotype and phenotype. (XLSX $100 \mathrm{~kb}$ )

Additional file 2: The sequence of Xbarc239. (TXT $1 \mathrm{~kb}$ )

\begin{abstract}
Abbreviations
QTL: Quantitative trait locus; TKW: Thousand kernel weight; SSR: Simple sequence repeats; MAS: Marker-assisted selection; PAGE: Polyacrylamide gel electrophoresis; IWGSC: International wheat genome sequencing consortium; SNP: Single nucleotide polymorphism; CIM: Composite interval mapping; LOD: Logarithm of the odds; CVs: Coefficients of variation; GFD: Grain filling duration; $h_{B}^{2}$ : Broad-sense heritability; Tmax: Daily maximum temperature.
\end{abstract}

\section{Competing interests}

The authors declare that they have no conflict of interests.

\section{Authors' contributions}

ZFN, QXS and XJC conceived the project; XJC conducted the field evaluations and TKW phenotyping with assistances from HJZ, AJZ and LX; $X J C$ carried out genotyping and sequencing experiments with assistances from LLC and ZYC. XJC analyzed the data with assistances from HRP, YYY and MSY; XJC and ZFN wrote the manuscript. All authors read and approved the final manuscript.

\section{Acknowledgments}

We thank Dr. Fengwu Zhao for providing Fu4185. This work was supported by the National Natural Science Foundation of China (91435204 and 31271710), the 863 Project Grant (2012AA10A309).

\section{Author details}

${ }^{1}$ State Key Laboratory for Agrobiotechnology and Key Laboratory of Crop Heterosis and Utilization (MOE) and Key Laboratory of Crop Genomics and Genetic Improvement (MOA), Beijing Key Laboratory of Crop Genetic Improvement, China Agricultural University, Yuanmingyuan Xi Road No. 2,
Haidian District, Beijing 100193, China. ${ }^{2}$ Department of Plant Genetics and Breeding, China Agricultural University, Beijing 100193, China. ${ }^{3}$ National Plant Gene Research Centre, Beijing 100193, China. ${ }^{4}$ Hebei Crop Genetic Breeding Laboratory Institute of Cereal and Oil Crops, Hebei Academy of Agriculture and Forestry Sciences, Shijiazhuang 050035, China.

Received: 22 July 2015 Accepted: 21 October 2015

Published online: 28 October 2015

\section{References}

1. Smith R. Increasing wheat yield requires genetics, management. Southwest Farm Press. http://southwestfarmpress.com/wheat/increasing-wheat-yieldrequires-genetics-management. Accessed 6 February 2014.

2. Peng JH, Ronin Y, Fahima T, Roder MS, Li YC, Nevo E, et al. Domestication quantitative trait loci in Triticum dicoccoides, the progenitor of wheat. Proc Natl Acad Sci U S A. 2003;100:2489-94.

3. Wang LF, Ge HM, Hao CY, Dong YS, Zhang XY. Identifying loci influencing 1,000-kernel weight in wheat by microsatellite screening for evidence of selection during breeding. PLoS One. 2012;7(2):e29432.

4. Campbell KG, Bergmem CJ, Gualberto DG, Anderson JA, Giroux MJ, Hareland $\mathrm{G}$, et al. Quantitative trait loci associated with kernel traits in a soft $\times$ hard wheat cross. Crop Sci. 1999:39:1184-95.

5. Campbell BT, Baenziger PS, Gill KS, Eskridge KM, Budak H, Erayman M, et al. Identification of QTLs and environmental interactions associated with agronomic traits on chromosome 3A wheat. Crop Sci. 2003:43:1493-505.

6. Kato K, Miura H, Sawada S. Mapping QTLs controlling grain yield and its components on chromosome 5A of wheat. Theor Appl Genet. 2000;101:1114-21.

7. Varshney RK, Prasad M, Roy JK, Kumar N, Singh H, Dhaliwal HS, et al. Identification of eight chromosomes and one microsatellite marker on 1AS associated with QTL for grain weight in bread wheat. Theor Appl Genet. 2000;100:1290-5.

8. Groos C, Robert N, Bervas E, Charmet G. Genetic analysis of grain proteincontent, grain yield and thousand-kernel weight in bread wheat. Theor Appl Genet. 2003;106:1032-40.

9. Kumar N, Kulwal PL, Gaur A, Tyagi AK, Khurana JP, Khurana P, et al. QTL analysis for grain weight in common wheat. Euphytica. 2006;151:135-44.

10. Jiang QY, Hou J, Hao CY, Wang LF, Ge HM, Dong YS, et al. The wheat (T. aestivum) sucrose synthase 2 gene (TaSus2) active in endosperm development is associated with yield traits. Funct Integr Genomics. 2001;11(1):49-61.

11. Su ZQ, Hao CY, Wang LF, Dong YC, Zhang XY. Identification and development of a functional marker of TaGW2 associated with grain weight in bread wheat (Triticum aestivum L.). Theor Appl Genet. 2011;122(1):211-23.

12. Zhang $L$, Zhao YL, Gao LF, Zhao GY, Zhou RH, Zhang BS, et al. TaCKX6-D1, the ortholog of rice OsCKX2, is associated with grain weight in hexaploid wheat. New Phytol. 2012;195(3):574-84.

13. Asseng S, Ewert F, Martre P, Rötter RP, Lobell DB, Cammarano D, et al. Rising temperatures reduce global wheat production. Nat Clim Change. 2015;5:143-7.

14. Reynolds M, Balota M, Delgado M, Amani I, Fischer R. Physiological and morphological traits associated with spring wheat yield under hot, irrigated conditions. Funct Plant Biol. 1994;21(6):717-30.

15. Wardlaw I, Wrigley C. Heat tolerance in temperate cereals: an overview. Funct Plant Biol. 1994;21(6):695-703

16. Sharma RC, Tiwari AK, Ortiz-Ferrara G. Reduction in kernel weight as a potential indirect selection criterion for wheat grain yield under heat stress. Plant Breed. 2008;127:241-8.

17. Pinto RS, Reynolds MP, Mclntyre CL, Olivares-Villegas JJ, Chapman SC. Heat and drought QTL in a wheat population designed to minimize confounding agronomic effects. Theor Appl Genet. 2010;121:1001-21.

18. Paliwal R, Röder MS, Kumar U, Srivastava JP, Joshi AK. QTL mapping of terminal heat tolerance in hexaploid wheat ( $T$. aestivum L.). Theor Appl Genet. 2012;125(3):561-75.

19. Pandey GC, Sareen S, Siwach P, Tiwari R. Molecular characterization of heat tolerance in bread wheat (Triticum aestivum L.) using differences in thousandgrain weights (dTGW) as a potential indirect selection criterion. Cereal Res Commun. 2014;1:38-46.

20. Sharp PJ, Kreis M, Shewry PR, Gale MD. Location of $\beta$-amylase sequences in wheat and its relatives. Theor Appl Genet. 1988;75(2):286-90. 
21. Marklund S, Chaudhary R, Marklund L, Sandberg K, Andersson L. Extensive mtDNA diversity in horses revealed by PCR-SSCP analysis. Anim Genet. 1995;26:193-6.

22. Brenchley R, Spannagl M, Pfeifer M, Barker GL, D'Amore R, Allen AM, et al. Analysis of the bread wheat genome using whole-genome shotgun sequencing. Nature. 2012;491(7426):705-10.

23. Luo MC, Gu YQ, You FM, Deal KR, Ma Y, Hu Y, et al. A 4-gigabase physical map unlocks the structure and evolution of the complex genome of Aegilops tauschii, the wheat D-genome progenitor. Proc Natl Acad Sci U S A. 2013;110(19):7940-5.

24. Wang S, Basten CJ, Zeng ZB. Windows QTL cartographer 2.5. Department of Statistics, North Carolina State University, Raleigh, NC.2012. http:// statgen.ncsu.edu/qtlcart/WQTLCart.htm. Accessed 01 Aug 2012.

25. Churchill GA, Doerge RW. Empirical threshold values for quantitative trait mapping. Genetics. 1994;138(3):963-71.

26. Doerge RW, Churchill GA. Permutation tests for multiple loci affecting a quantitative character. Genetics. 1996;142:285-94.

27. Yang J, Bai G, Shaner GE. Novel quantitative trait loci (QTL) for Fusarium head blight resistance in wheat cultivar Chokwang. Theor Appl Genet. 2005;111(8):1571-9.

28. Vigouroux Y, Jaqueth JS, Matsuoka Y, Smith OS, Beavis WD, Smith JS, et al Rate and pattern of mutation at microsatellite loci in maize. Mol Biol Evol. 2002;19(8):1251-60.

29. Schug MD, Mackay TF, Aquadro CF. Low mutation rates of microsatellite loci in Drosophila melanogaster. Nat Genet. 1997;15(1):99-102.

30. Schlötterer C, Ritter R, Harr B, Brem G. High mutation rate of a long microsatellite allele in Drosophila melanogaster provides evidence for allelespecific mutation rates. Mol Biol Evol. 1998;1510:1269-74.

31. Schug MD, Hutter CM, Wetterstrand KA, Gaudette MS, Mackay TF, Aquadro CF. The mutation rates of di-, tri- and tetranucleotide repeats in Drosophila melanogaster. Mol Biol Evol. 1998;15(12):1751-60.

32. Brinkmann B, Klintschar M, Neuhuber $F$, Hühne J, Rolf B. Mutation rate in human microsatellites: influence of the structure and length of the tandem repeat. Am J Hum Genet. 1998;62(6):1408-15.

33. Xu X, Peng M, Fang Z. The direction of microsatellite mutations is dependent upon allele length. Nat Genet. 2000;24(4):396-9.

34. Morita R, Kusaba M, lida S, Yamaguchi H, Nishio T, Nishimura M. Molecular characterization of mutations induced by gamma irradiation in rice. Genes Genet Syst. 2009;84(5):361-70.

35. Somers DJ, Isaac P, Edwards K. A high-density microsatellite consensus map for bread wheat (Triticum aestivum L.). Theor Appl Genet. 2004;109(6):1105-14.

36. Lynch M. Evolution of the mutation rate. Trends Genet. 2010;26(8):345-52.

37. Nesbitt M, Samuel D. From staple crop to extinction? The archaeology and history of the hulled wheats. In S. Padulosi, K. Hammer, J. Heller, editors. In Proceedings of the First International Workshop on Hulled Wheats: 21-22 July 1995. Italy: Tuscany, Castelvecchio Pascoli; 1995. pp. 41-102.

38. Petersen G, Seberg O, Yde M, Berthelsen K. Phylogenetic relationships of Triticum and Aegilops and evidence for the origin of the $A, B$, and $D$ genomes of common wheat (Triticum aestivum). Mol Phylogenet Evol. 2006:39:70-82

39. Tsunewaki K. Comparative gene analysis of common wheat and its ancestral species. II. Waxiness, growth habit and awnedness. Jpn J Bot. 1966;19:175-229.

40. Dvorak J, Luo MC, Yang ZL, Zhang HB. The structure of the Aegilops tauschii genepool and the evolution of hexaploid wheat. Theor Appl Genet. 1998;97:657-70.

41. Feldman M, Origin of cultivated wheat. In: Bonjean AP, Angus WJ, editors. The world wheat book: a history of wheat breeding. Paris: rue Lavoisier; 2001. p. 3-53.

42. Dubcovsky J, Dvorak J. Genome plasticity a key factor in the success of polyploid wheat under domestication. Science. 2007;316:1862-6.

43. Naghavi MR, Aghaei MJ, Taleei AR, Omidi M, Mozafari J, Hassani ME. Genetic diversity of the D-genome in T. aestivum and Aegilops species using SSR markers. Genet Resour Crop Evol. 2009;56:499-506.

44. Montaldo HH, Meza-Herrera CA. Use of molecular markers and major genes in the genetic improvement of livestock. Electron J Biotechnol. 1998;2:15-6.

45. Simmonds J, Scott P, Leverington-Waite M, Turner AS, Brinton J, Korzun V, et al. Identification and independent validation of a stable yield and thousand grain weight QTL on chromosome 6A of hexaploid wheat (Triticum aestivum L.). BMC Plant Biol. 2014;14(1):191.
46. Talukder SK, Babar MA, Vijayalakshmi K, Poland J, Prasad PW, Bowden R, et al. Mapping QTL for the traits associated with heat tolerance in wheat (Triticum aestivum L.). BMC Genet. 2014;15(1):97.

47. Yang J, Sears RG, Gill BS, Paulsen GM. Quantitative and molecular characterization of heat tolerance in hexaploid wheat. Euphytica. 2002;126:275-82.

48. Vargas M, van Eeuwijk FA, Crossa J, Ribaut JM. Mapping QTLs and QTL x environment interaction for CIMMYT maize drought stress program using factorial regression and partial least squares methods. Theor Appl Genet. 2006;112(6):1009-23.

49. Collins NC, Tardieu F, Tuberosa R. Quantitative trait loci and crop performance under abiotic stress: where do we stand? Plant Physiol. 2008;147(2):469-86

50. Ge H, You G, Wang L, Hao CY, Dong YS, Li ZS, et al. Genome selection sweep and association analysis shed light on future breeding by design in wheat. Crop Sci. 2012;52(3):1218-28.

51. Sun XY, Wu K, Zhao Y, Kong FM, Han GZ, Jiang HM, et al. QTL analysis of kernel shape and weight using recombinant inbred lines in wheat. Euphytica. 2009;165(3):615-24.

52. Wang Y, Hao C, Zheng J, Ge HM, Zhou Y, Ma ZQ et al. A haplotype block associated with thousand-kernel weight on chromosome 5DS in common wheat (Triticum aestivum L.). J Integr Plant Biol. 2014;57(8):662-72.

53. Okamoto $Y$, Nguyen AT, Yoshioka M, lehisa JC, Takumi S. Identification of quantitative trait loci controlling grain size and shape in the $D$ genome of synthetic hexaploid wheat lines. Breed Sci. 2013;63(4):423-9.

\section{Submit your next manuscript to BioMed Central and take full advantage of:}

- Convenient online submission

- Thorough peer review

- No space constraints or color figure charges

- Immediate publication on acceptance

- Inclusion in PubMed, CAS, Scopus and Google Scholar

- Research which is freely available for redistribution 